\section{Conclusions and perspectives}

The current reports by Rentschler et al. and Aanhaanen et al. clearly demonstrate that accessory pathway formation underlying ventricular preexcitation arises as a consequence of erroneous development of the AV canal myocardium. A hierarchical network of transcription factors, including Tbx2/3/5, Nkx2.5, GATA4, and Msx2, governs normal development of the AV canal under the regulation of Notch and BMP signaling (10). Contrary to previous reports demonstrating that Notch signaling restricts BMP2 and $\mathrm{Tb} \times 2 / 3$ to the $\mathrm{AV}$ canal during early embryogenesis $(16,17)$, the expression level of BMP2 was unchanged in Notch1-activated mice (1). Our understanding of the pathophysiology of ventricular preexcitation will be broadened by further characterization of the genetic program for AV canal development.

\section{Acknowledgments}

This work was supported in part by grants from Japan Society for the Promotion of Science (KAKENHI 20390218, 21229010); Health and Labor Sciences Research grants (to I. Komuro and H. Akazawa); and grants from Astellas Foundation for Research on Metabolic Disorders, The Uehara Memorial Foundation, The Ichiro Kanehara Foundation, Mochida Memorial Foundation for Medical and Pharmaceutical Research, and Suzuken Memorial Foundation (to H. Akazawa).
Address correspondence to: Issei Komuro, Department of Cardiovascular Medicine, Osaka University Graduate School of Medicine, 2-2 Yamadaoka, Suita, Osaka 565-0871, Japan. Phone: 81.6.6879.3631; Fax: 81.6.6879.3639; E-mail: komuro-tky@ umin.ac.jp.

1. Rentschler $S$, et al. Notch signaling regulates murine atrioventricular conduction and the formation of accessory pathways. J Clin Invest. 2011;121(2):525-533.

2. Aanhaanen WTJ, et al. Defective Tbx2-dependent patterning of the atrioventricular canal myocardium causes accessory pathway formation in mice. J Clin Invest. 2011;121(2):534-544.

3. Blair E, et al. Mutations in the gamma(2) subunit of AMP-activated protein kinase cause familial hypertrophic cardiomyopathy: evidence for the central role of energy compromise in disease pathogenesis. Hum Mol Genet. 2001;10(11):1215-1220.

4. Gollob $\mathrm{MH}$, et al. Identification of a gene responsible for familial Wolff-Parkinson-White syndrome. N Engl J Med. 2001;344(24):1823-1831.

5. Arad M, Seidman CE, Seidman JG. AMP-activated protein kinase in the heart: role during health and disease. Circ Res. 2007;100(4):474-488.

6. Arad M, et al. Transgenic mice overexpressing mutant PRKAG2 define the cause of Wolff-Parkinson-White syndrome in glycogen storage cardiomyopathy. Circulation. 2003;107(22):2850-2856.

7. Sidhu JS, et al. Transgenic mouse model of ventricular preexcitation and atrioventricular reentrant tachycardia induced by an AMP-activated protein kinase loss-of-function mutation responsible for Wolff-Parkinson-White syndrome. Circulation. 2005;111(1):21-29.

8. Wessels A, Markman MW, Vermeulen JL, Anderson RH, Moorman AF, Lamers WH. The development of the atrioventricular junction in the human heart. Circ Res. 1996;78(1):110-117.

9. Kolditz DP, et al. Epicardium-derived cells in development of annulus fibrosis and persistence of acces- sory pathways. Circulation. 2008;117(12):1508-1517.

10. Hatcher CJ, Basson CT. Specification of the cardiac conduction system by transcription factors. Circ Res. 2009;105(7):620-630.

11. Aanhaanen WT, et al. The Tbx $2+$ primary myocardium of the atrioventricular canal forms the atrioventricular node and the base of the left ventricle. Circ Res. 2009;104(11):1267-1274.

12. Aanhaanen WT, et al. Developmental origin, growth, and three-dimensional architecture of the atrioventricular conduction axis of the mouse heart. Circ Res. 2010;107(6):728-736.

13. Tallini YN, et al. Imaging cellular signals in the heart in vivo: Cardiac expression of the high-signal Ca2+ indicator GCaMP2. Proc Natl Acad Sci U S A. 2006;103(12):4753-4758.

14. Kolditz DP, et al. Persistence of functional atrioventricular accessory pathways in postseptated embryonic avian hearts: implications for morphogenesis and functional maturation of the cardiac conduction system. Circulation. 2007;115(1):17-26.

15. Hahurij ND, et al. Accessory atrioventricular myocardial connections in the developing human heart: relevance for perinatal supraventricular tachycardias. Circulation. 2008;117(22):2850-2858.

16. Rutenberg JB, Fischer A, Jia H, Gessler M, Zhong TP, Mercola M. Developmental patterning of the cardiac atrioventricular canal by Notch and Hairy-related transcription factors. Development. 2006;133(21):4381-4390.

17. Kokubo H, Tomita-Miyagawa S, Hamada Y, Saga Y. Hesr 1 and Hesr2 regulate atrioventricular boundary formation in the developing heart through the repression of Tbx2. Development. 2007;134(4):747-755.

18. Ma L, Lu MF, Schwartz RJ, Martin JF. Bmp2 is essential for cardiac cushion epithelial-mesenchymal transition and myocardial patterning. Development. 2005;132(24):5601-5611.

19. Harrelson $Z$, et al. Tbx2 is essential for patterning the atrioventricular canal and for morphogenesis of the outflow tract during heart development. Development. 2004;131(20):5041-5052.

20. Anderson RH, Ho SY. Anatomy of the atrioventricular junctions with regard to ventricular preexcitation. Pacing Clin Electrophysiol. 1997;20(8 pt 2):2072-2076.

\title{
Growing a tumor stroma: a role for granulin and the bone marrow
}

Andrew Bateman

Endocrine Research Laboratory, Royal Victoria Hospital, Research Institute of the McGill University Health Centre, Montreal, Quebec, Canada.

\begin{abstract}
The tumor stroma is critical in cancer progression; understanding its formation is therefore important biologically and therapeutically. In this issue of the JCI, Elkabets et al. report on the generation of data in mice that lead them to propose that certain tumors can stimulate the growth of a second otherwise quiescent or indolent tumor in the same animal by stimulating stromal formation. Granulin-expressing $\mathrm{Sca}^{+} \mathrm{Kit}^{-}$hematopoietic progenitor cells in the bone marrow of the tumor host were required to mediate this effect. These data shed new light on the importance of the bone marrow in tumor growth and the role of granulin in carcinogenesis.
\end{abstract}

Conflict of interest: The author has declared equity in Neurodyn Inc.

Citation for this article: J Clin Invest. 2011;

121(2):516-519. doi:10.1172/JCI46088.

\section{Background: systemic tumor instigation}

The reactive (or desmoplastic) stroma is an aberrant fibrous tissue that surrounds can- cer cells (1). It is formed from fibroblasts, adipocytes, inflammatory cells, and vascular cells and is further characterized by the presence of myofibroblasts (1). Myofibroblasts display properties not usually associated with fibroblasts in healthy tissue, such as the expression of $\alpha \mathrm{SMA}$, and secrete high levels of matrix proteins such as collagen I (1). The molecular properties of tumor stroma are predictive of disease outcome (2), with the stromal cells, in particular the myofibroblasts, stimulating tumor growth, invasion, and metastasis (1). For example, molecular crosstalk between cancer cells 


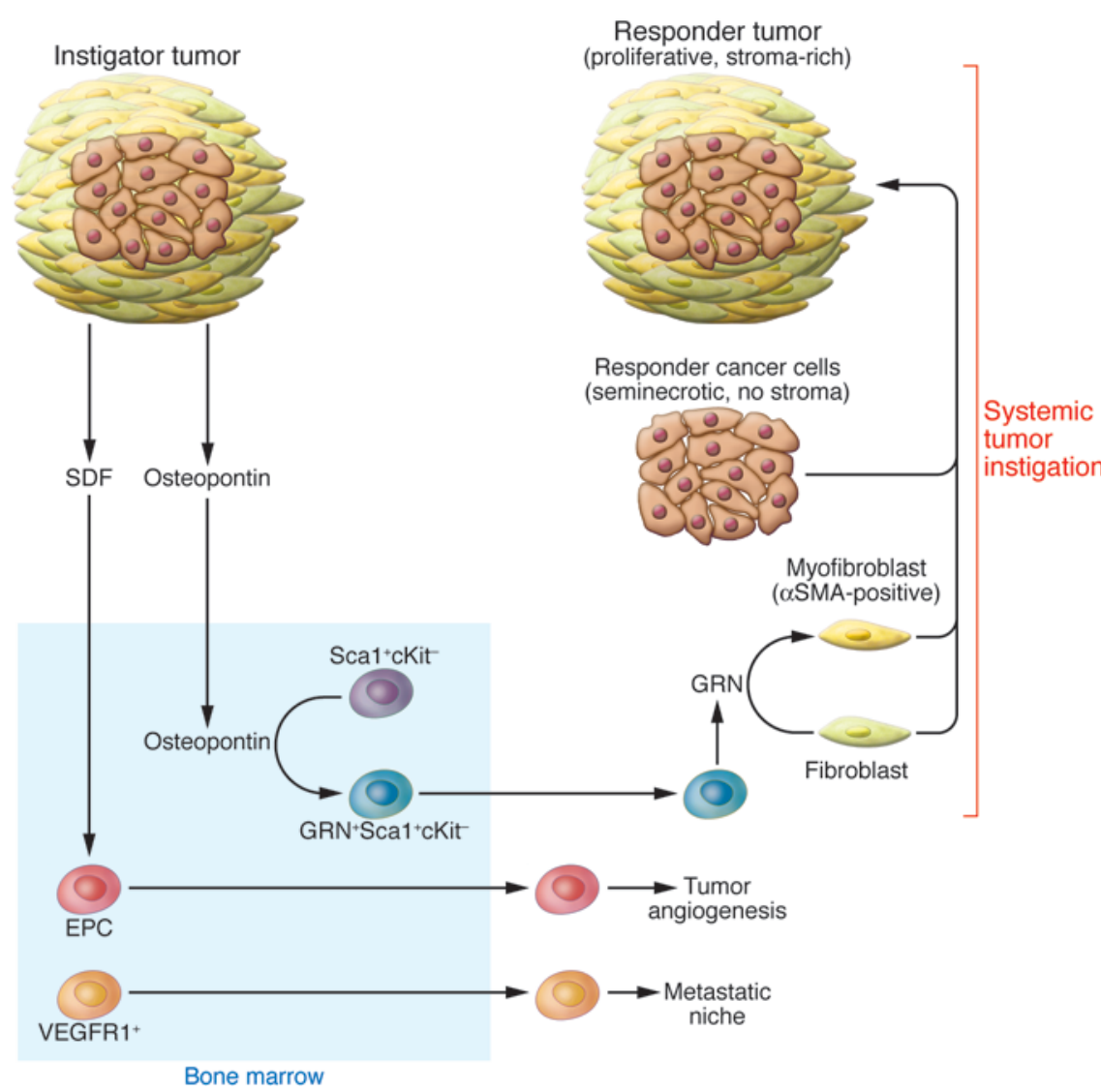

Figure 1

The role of GRN + Sca-1+cKit- BMCs in systemic instigation and the formation of tumor stroma. Cells from an aggressively growing primary tumor, the instigator, secrete the circulating signal osteopontin. This mobilizes a population of Sca- $1^{+} \mathrm{CKit}-\mathrm{BM}$ progenitor cells that express high levels of the secreted glycoprotein GRN. The GRN ${ }^{+}$Sca- $1^{+}{ }^{+}$Kit $B M C s$ travel to the site of inoculation of a second cancer cell type, the responder, that grows poorly in mice. GRN ${ }^{+} \mathrm{Sca}-1^{+} \mathrm{CKit}^{-}$ cells enhance the assembly of the tumor stoma by stimulating the differentiation of fibroblasts into $\alpha$ SMA-expressing myofibroblasts. The stroma, in turn, supports the successful growth of the otherwise quiescent or indolent responder cells, resulting in a proliferating stroma-rich carcinoma outgrowth (7). The process whereby an aggressive primary tumor promotes the outgrowth of another otherwise indolent tumor is called systemic instigation. Interrupting the paracrine GRN signal during systemic instigation or preventing the mobilization of the $\mathrm{GRN}^{+} \mathrm{Sca}-1^{+} \mathrm{CKit}^{-}$ BMCs might prove therapeutically useful in preventing the formation of reactive tumor stroma, thereby inhibiting tumor progression (7). The role of $\mathrm{GRN}+\mathrm{Sca}-1^{+} \mathrm{CKit}-\mathrm{BMCs}$ as local regulators of tumor progression is compared to other proposed mechanisms of $\mathrm{BM} /$ tumor interaction. For example, the BM may provide progenitor cells directly; in the example shown, tumor fibroblasts secrete stromal-derived factor-1 (SDF), which mobilizes endothelial progenitor cells (EPC). These contribute to angiogenesis (16). Other hematopoietic progenitors, for example VEGFR $1^{+}$ cells, may assist in the creation of prometastatic niches (17).

and myofibroblasts, mostly in the form of growth factor signaling $(1,3)$, promotes invasive growth of cancer cells $(1,3)$.

Several mechanisms have been put forward to explain how the tumor stroma forms, including through the recruitment and differentiation of cells in or near the tumor (1), through the recruitment into the tumor of circulating BM-derived cells, such as mesenchymal stem cells and fibrocytes $(1,4)$, and through epithelial-mesenchymal transition of cancer cells (1). A previous study by McAllister, Weinberg, and colleagues (5) defined in mice an additional mechanism that they called systemic instigation. Underpinning this mechanism is the concept that a circulating signal is secreted from an aggressively tumor-forming breast cancer cell line, which is called the instigator tumor, that results in tumor outgrowth and the acquisition of a reactive stroma in a second otherwise quiescent or indolent breast cancer cell line, known as the responder. If the instigator tumor is absent, the responder cells fail to expand and may even become necrotic (Figure 1).

Systemic instigation acts through BM cells (BMCs). The instigator tumor secretes the prometastatic factor osteopontin (6), which then mobilizes $\mathrm{Sca} 1^{+} \mathrm{cKit}{ }^{-}$cells from the BM. The $\mathrm{Sca}^{+} \mathrm{CKit}^{-} \mathrm{BMCs}$ accumulate at the responder tumor and stimulate reactive stroma formation (ref. 5 and Figure 1). However, how these $\mathrm{Sca} 1^{+} \mathrm{cKit}^{-} \mathrm{BMCs}$ stimulate formation of a reactive stroma has not been defined (5). This point has now been addressed in this issue of the JCI, where Elkabets et al. (7) report for the first time the mechanism by which $\mathrm{Sca} 1^{+} \mathrm{CKit}^{-} \mathrm{BMCs}$ instigate the formation of a reactive stroma in indolent responder tumors in mice.

\section{GRN+Sca1+cKit-CD45+ cells mediate systemic instigation}

To follow the fate of BMCs during tumor instigation, Elkabets and colleagues performed BM transplants using donor marrow that expressed GFP (7). As expected, $\mathrm{GFP}^{+} \mathrm{BMCs}$ were recruited to the stroma of responder tumors. However, very few $\mathrm{GFP}^{+}$myofibroblasts were observed in the responder tumor stroma. It was deemed unlikely therefore that $\mathrm{Sca} 1^{+} \mathrm{cKit}^{-} \mathrm{BMCs}$ were stromal progenitor cells; rather it seemed more likely that they acted in a regulatory capacity to coordinate the assembly of the stroma from other cell types. BMCs from mice with instigator tumors were sorted into $\mathrm{Sca} 1^{+} \mathrm{cKit}^{-}, \mathrm{Sca}{ }^{+}{ }^{+} \mathrm{Kit}^{+}$, and Sca $1^{-}$cells and comixed with responder cancer cells before transplantation into mice that did not bear contralateral instigator tumors. Of these three cellular subsets, only Sca ${ }^{+} \mathrm{CKit}^{-}$cells reproduced the effects of an instigator tumor, enabling responder tumors to elaborate a collagen-rich stroma with $\alpha \mathrm{SMA}^{+}$cells. In contrast, $\mathrm{Sca}^{+}{ }^{+} \mathrm{Kit}^{-}$ cells that were harvested from the BM of control mice with either Matrigel or an aggressive tumor that did not instigate the responder tumor in place of the instigator tumor were unable to promote formation of a reactive stroma (7).

$\mathrm{Sca} 1^{+} \mathrm{CKit}^{-}$cells are a subclass of quiescent hematopoietic progenitor cells of illdefined function present in all mouse BM (8). Elkabets et al. (7) therefore asked in what way stroma-instigating $\mathrm{Sca} 1^{+} \mathrm{cKit}^{-} \mathrm{BMCs}$ differed from inactive control Sca $1^{+} \mathrm{CKit}^{-}$ BMCs. No differences were observed in cell-surface markers, with both instigator and control Sca $1^{+} \mathrm{CKit}^{-}$BMCs displaying 
the hematopoietic marker CD45. However, microarray analyses revealed that instigator $\mathrm{Sca} 1^{+} \mathrm{CKit}-\mathrm{BMCs}$ expressed greater levels of the Grn gene, which encodes the secreted protein Granulin (GRN; sometimes called progranulin, PC-derived growth factor, granulin-epithelin precursor, proepithelin, acrogranin, or TGF-e) (9). Importantly tumor-resident BMCs in responder tumors grown opposite instigator tumors were also $\mathrm{GRN}^{+}$, whereas $\alpha \mathrm{SMA}^{+}$myofibroblasts and cancer cells were GRN'-

Elkabets et al. (7) then investigated whether GRN might mediate stroma instigation by the $\mathrm{GRN}^{+} \mathrm{Sca} 1^{+} \mathrm{cKit}-\mathrm{CD} 45^{+}$BMCs. In vivo stimulation of indolent tumors with recombinant GRN fully recapitulated the effect of instigator tumors, promoting the formation of responder tumor foci with an adenocarcinoma-like morphology. GRN induced the differentiation of cultured human mammary fibroblasts into $\alpha \mathrm{SMA}^{+}$ myofibroblast-like cells. Responder cancer cells in culture did not proliferate in response to GRN. This suggests that during systemic instigation, the proliferation of responder cancer epithelial cells is not stimulated directly by GRN but likely results secondarily through the increased formation of a reactive stroma. In agreement with this hypothesis, transplantation into mice that lacked instigator tumors of responder cells comixed with normal primary mammary fibroblasts primed in vitro with GRN resulted in the growth of proliferative foci of responder tumor cells (7). Elkabets et al. (7) therefore concluded that $\mathrm{GRN}^{+} \mathrm{Sca} 1^{+} \mathrm{CKit}^{-} \mathrm{CD} 45^{+}$BMCs mediate systemic instigation of tumor stroma through the secretion of GRN.

\section{Progranulin in human breast cancers}

In mice, $\mathrm{GRN}^{+}$cells in both responder and instigator tumors were found only in the stroma (7), suggesting tentatively that instigator tumors may instigate the formation of their own stromal tissue. Although stromal cells in human breast cancers express GRN, Elkabets et al. (7) observed that GRN was also expressed in human cancer epithelial cells (7). They further observed a positive correlation between GRN expression and aggressive, treatment-resistant, triple-negative breast cancers (that is, breast cancers that lack the receptors for estrogen and progesterone, as well as Her2/neu). GRN-positivity also correlated with the size of the tumor and its proliferative index. Kaplan-Meier analyses indicated a relationship between
GRN positivity of breast cancers and worse survival among patients (7). These studies did not differentiate between stromal and epithelial expression of GRN.

The triple-negative state of a breast cancer refers to the lack of expression of the three receptors on the cancerous cells of epithelial origin, not the stroma. Epithelial GRN expression has been associated previously with advanced invasive cancers (9, 10). In previous reports in mice, depleting GRN from cancer cells by mRNA targeting (11) or monoclonal antibody strategies (12) reduced their tumorigenicity, while overexpressing GRN in cells that are only weakly tumorigenic enhanced their malignant properties $(13,14)$. Taken together, these data suggest a model in which GRN delivered to a tumor by $\mathrm{GRN}^{+} \mathrm{Sca} 1^{+} \mathrm{CKit}^{-} \mathrm{CD} 45^{+}$ BMCs plays a key role in the early stages of tumor development, particularly in stromal formation (7), but as tumors progress, GRN expression is no longer restricted to these cells (7), as cancer epithelial cells acquire the ability to both express and respond to GRN. Fibroblasts in wounds respond to GRN by increased proliferation and migration (15). GRN from cancer epithelial cells may therefore play a similar role in regulating fibroblast activity in the tumor stroma, perhaps even taking over part of the function of $\mathrm{GRN}^{+} \mathrm{Sca}^{+} \mathrm{cKit}^{-}$ $\mathrm{CD} 45^{+} \mathrm{BMCs}$, although at this stage that is purely speculative.

\section{Context and questions}

BMCs have been implicated extensively in tumor progression and invasion (Figure 1), primarily as the source of myofibroblast precursor cells for the tumor stroma $(1,4)$ or as endothelial progenitor cells that may be mobilized from the BM and recruited to tumors by tumor-derived chemokines such as stromal-derived factor-1 (16). Nevertheless, the mechanism for stroma formation proposed by Elkabets et al. (7) presents unique features, notably that the $\mathrm{GRN}^{+} \mathrm{Sca} 1^{+} \mathrm{CKit}^{-} \mathrm{CD} 45^{+} \mathrm{BMCs}$ recruited to the tumor direct the assembly of the reactive stroma but do not act themselves as precursors for stromal cells. Further work is needed to understand when systemic instigation, working through $\mathrm{GRN}^{+} \mathrm{Sca}{ }^{+}{ }^{+} \mathrm{CKit}^{-} \mathrm{CD} 45^{+} \mathrm{BMCs}$, contributes to stroma formation and when circulating progenitor cells from the BM are more likely to support the production of a reactive tumor stroma. BMCs promote metastasis by providing favorable local microenvironments in which metastasizing cancer cells assemble and grow, as exemplified by the formation of prometastatic niches by $\mathrm{VEGFR}^{+} \mathrm{BMCs}(17)$. Sca ${ }^{+} \mathrm{cKit}-$ cells, which are presumably identical or closely related to the $\mathrm{GRN}^{+} \mathrm{Sca}^{+}{ }^{+} \mathrm{CKit}^{-} \mathrm{CD} 45^{+}$cells of Elkabets et al. (7), are effective instigators of indolent breast cancer metastasis in mouse lungs (5). Clearly, the role of GRN and $\mathrm{GRN}^{+} \mathrm{Sca}{ }^{+} \mathrm{CKit}^{-} \mathrm{CD} 45^{+}$BMCs in metastasis warrants further research.

As with all novel mechanisms, there are caveats in that suggested by the work of Elkabets et al. (7). Not all aggressively growing primary tumors are instigators of indolent tumors (5). Furthermore, primary tumor resection often promotes the metastasis of distant dormant tumors (18). This is the opposite of what would be predicted from the model of systemic instigation as proposed by Elkabets et al. (7), and it will be important to learn what factors determine why primary tumors can act either as instigators (7) or inhibitors (18) of dormant tumors. Integrating the responder/instigator mechanism definitively into the natural history of cancer progression is clearly an important future goal.

\section{Conclusions}

The findings reported in this issue of the JCI by Elkabets et al. (7) on the mechanisms by which BMCs regulate the formation of a reactive tumor stroma during systemic instigation enrich our understanding of the interplay between BMCs and cancer; they also reinforce the importance of the tumor stroma in cancer progression and highlight new roles of GRN in tumor growth. This clearly raises the prospect that strategies targeting GRN or the $\mathrm{GRN}^{+} \mathrm{Sca}^{+} \mathrm{cKit}^{-}$ $\mathrm{CD} 45^{+}$BMCs might provide novel therapeutic approaches to blocking, or even reversing, stromal expansion in cancer and that by so doing they might slow or halt the growth of human tumors.

\section{Acknowledgments}

Work from the author's laboratory was supported by funds from Canadian Institutes of Health Research and the Canadian Cancer Society Research Institute.

Address correspondence to: Andrew Bateman, Endocrine Research Laboratory, Room H.5.21 Royal Victoria Hospital, Research Institute of the McGill University Health Centre, 687 Pine Ave. West, Montreal, Quebec H3A1A1, Canada. Phone: 514.934.1934; Fax: 514.843.2819; E-mail: Andrew.bateman@muhc.mcgill.ca. 
1. De Wever O, Demetter P, Mareel M, Bracke M Stromal myofibroblasts are drivers of invasive cancer growth. Int J Cancer. 2008;123(10):2229-2238.

2. Finak G, et al. Stromal gene expression predicts clinical outcome in breast cancer. Nat Med. 2008; 14(5):518-527.

3. Bhowmick NA, Moses HL. Tumor-stroma interactions. Curr Opin Genet Dev. 2005;15(1):97-101.

4. Ishii $\mathrm{G}$, et al. Bone-marrow-derived myofibroblasts contribute to the cancer-induced stromal reaction. Biochem Biophys Res Commun. 2003;309(1):232-240.

5. McAllister SS, et al. Systemic endocrine instigation of indolent tumor growth requires osteopontin. Cell. 2008;133(6):994-1005.

6. Anborgh PH, Mutrie JC, Tuck AB, Chambers AF. Role of the metastasis-promoting protein osteopontin in the tumour microenvironment. J Cell Mol Med. 2010;14(8):2037-2044.

7. Elkabets $\mathrm{M}$, et al. Human tumors instigate granulin-expressing hematopoietic cells that promote malignancy by activating stromal fibroblasts in mice. J Clin Invest. 2011;121(2):784-799.

8. Randall TD, Weissman IL. Characterization of a population of cells in the bone marrow that phenotypically mimics hematopoietic stem cells: Resting cells or mystery population? Stem Cells. 1998;16(1):38-48.

9. Bateman A, Bennett HP. The granulin gene family: from cancer to dementia. Bioessays. 2009; 31(11):1245-1254.

10. Serrero G, Ioffe OB. Expression of PC-cell-derived growth factor in benign and malignant human breast epithelium. Hum Pathol. 2003;34(11):1148-1154.

11. Lu R, Serrero G. Inhibition of PC cell-derived growth factor (PCDGF, epithelin/granulin precursor) expression by antisense PCDGF cDNA transfection inhibits tumorigenicity of the human breast carcinoma cell line MDA-MB-468. Proc Natl Acad Sci U S A. 2000;97(8):3993-3998.

12. Ho JC, et al. Granulin-epithelin precursor as a therapeutic target for hepatocellular carcinoma. Hepatology. 2008;47(5):1524-1532.

13. He Z, Bateman A. Progranulin gene expres- sion regulates epithelial cell growth and promotes tumor growth in vivo. Cancer Res. 1999; 59(13):3222-3229.

14. Miyanishi $M$, et al. Immortalized ovarian surface epithelial cells acquire tumorigenicity by Acrogranin gene overexpression. Oncol Rep. 2007; 17(2):329-333.

15. He Z, Ong CH, Halper J, Bateman A. Progranulin is a mediator of the wound response. Nat Med. 2003;9(2):225-229.

16. Orimo A, et al. Stromal fibroblasts present in invasive human breast carcinomas promote tumor growth and angiogenesis through elevated SDF-1/ CXCL12 secretion. Cell. 2005;121(3):335-348.

17. Kaplan RN, et al. VEGFR1-positive haematopoietic bone marrow progenitors initiate the pre-metastatic niche. Nature. 2005;438(7069):820-827.

18. Holmgren L, O'Reilly MS, Folkman J. Dormancy of micrometastases: balanced proliferation and apoptosis in the presence of angiogenesis suppression. Nat Med. 1995;1(2):149-153.

\title{
Nuclear receptors take center stage in Th17 cell-mediated autoimmunity
}

\author{
Jennifer J. Heller, Ju Qiu, and Liang Zhou
}

Department of Pathology and Department of Microbiology and Immunology, Feinberg School of Medicine, Northwestern University, Chicago, Illinois, USA.

\begin{abstract}
Liver $X$ receptors (LXRs) are nuclear receptors involved in cholesterol homeostasis. Notably, they are also expressed by $T$ cells and are involved in regulating $\mathrm{T}$ cell proliferation and differentiation. In this issue of the JCI, Cui et al. have elucidated the molecular mechanism underlying the effects of LXR activation on a subset of T cells known as Th17 cells in mice and humans. Specifically, they showed that LXR-induced Srebp-1 inhibits Il17 transcription by binding to the Il17 promoter through interaction with the aryl hydrocarbon receptor (Ahr), a transcription factor known to enhance Th17 cell responses.
\end{abstract}

Heightened immune responses are needed to fight harmful microorganisms, yet those same responses have to be kept from overreacting and attacking the body's normal tissues to cause autoimmunity. This balance is maintained by a complex set of interactions among various immune cells. Th17 cells are a subset of $\mathrm{CD} 4^{+} \mathrm{T}$ cells characterized by the production of high levels of the cytokines IL-17 (also known as IL-17A), IL-17F, and IL-22 (1). They are important for the clearance of certain bacterial and fungal pathogens (1). However, Th17 cells have also been implicated in various human autoimmune diseases and other chronic inflammatory conditions (1). Since the initial discovery that the nuclear retinoic

Conflict of interest: The authors have declared that no conflict of interest exists.

Citation for this article: J Clin Invest. 2011;

121(2):519-521. doi:10.1172/JCI45939. acid-related orphan receptor $\gamma \mathrm{t}(\mathrm{ROR} \gamma \mathrm{t})$ is key to the differentiation of Th17 cells (2), transcriptional regulation of Th17 cells has been intensively investigated.

\section{Aryl hydrocarbon receptor, an important regulator of Th17 cell differentiation}

Aryl hydrocarbon receptor (Ahr), a liganddependent nuclear receptor, is among the handful of transcription factors implicated thus far in Th17 cell differentiation (3). Although its endogenous ligands remain elusive, Ahr is best known to mediate the effects of environmental toxins (e.g., the human carcinogen dioxin; ref. 4). Ahr belongs to the basic region helix-loop-helix (bHLH)/Per-Arnt-Sim (PAS) family of proteins (4). Ligand binding to the PAS domain triggers the transcriptional activity of Ahr, inducing target gene expression. Ligandactivated Ahr enhances the expression of IL-17 and is essential for IL-22 expression (5). Recent data suggest that Ahr may promote Th17 cell differentiation, in part through suppression of Stat1 phosphorylation (6). However, the precise molecular mechanism(s) by which Ahr regulates Th17 cell differentiation still remain elusive.

Role of liver $\mathrm{X}$ receptors in regulating immune response

Liver $\mathrm{X}$ receptors (LXRs) were originally classified as orphan nuclear receptors. However, it was subsequently determined that both LXR isoforms (LXR $\alpha$ and LXR $\beta$ ) are ligand-dependent transcription factors activated by metabolites of cholesterol that cause a conformational change in the LXR upon binding (7). LXRs form a heterodimer with retinoid $\mathrm{X}$ receptor (RXR), which then binds DNA and modulates the expression of various genes involved in regulating cholesterol homeostasis, including $A B C A 1, A B C G 1$, and SREBP1 (7). More recent studies have expanded the role of LXRs to implicate them as modulators of the immune response (8-11). LXR ligands have been shown to inhibit LPSor cytokine-induced expression of genes encoding inflammatory mediators such as IL-6 (8), and LXR-dependent gene expression is required for macrophage function in response to bacterial infection (9). 